\title{
Estimated pretreatment hemodynamic prognostic factors of aneurysm recurrence after endovascular embolization
}

\author{
Kouichi Misaki ${ }^{a}$, Hiroyuki Takao a,b,c,*, Takashi Suzuki ${ }^{a, b}$, Kengo Nishimura $^{\mathrm{a}}$, Issei Kan ${ }^{\mathrm{a}}$, \\ Ichiro Yuki ${ }^{\mathrm{a}}$, Toshihiro Ishibashi ${ }^{\mathrm{a}}$, Makoto Yamamoto $^{\mathrm{d}}$ and Yuichi Murayama ${ }^{\mathrm{a}}$ \\ ${ }^{a}$ Department of Neurosurgery, The Jikei University School of Medicine, Tokyo 105-8461, Japan \\ ${ }^{\mathrm{b}}$ Department of Innovation for Medical Information Technology, Jikei University School of Medicine, \\ Tokyo 105-8461, Japan \\ ${ }^{\mathrm{c}}$ Graduate School of Mechanical Engineering, Tokyo University of Science, Tokyo 125-8585, Japan \\ ${ }^{\mathrm{d}}$ Department of Mechanical Engineering, Tokyo University of Science, Tokyo 125-8585, Japan
}

Received 2 April 2017

Accepted 29 April 2017

\begin{abstract}
.
BACKGROUND: Hemodynamic factors play important roles in aneurysm recurrence after endovascular treatment.

OBJECTIVE: Predicting the risk of recurrence by hemodynamic analysis using an untreated aneurysm model is important because such prediction is required before treatment.

METHODS: We retrospectively analyzed hemodynamic factors associated with aneurysm recurrence from pretreatment models of five recurrent and five stable posterior communicating artery (Pcom) aneurysms with no significant differences in aneurysm volume, coil packing density, or sizes of the dome, neck, or Pcom. Hemodynamic factors of velocity ratio, flow rate, pressure ratio, and wall shear stress were investigated.

RESULTS: Among the hemodynamic factors investigated, velocity ratio and flow rate of the Pcom showed significant differences between the recurrence group and stable group $(0.630 \pm 0.062$ and $0.926 \pm 0.051, P=0.016 ; 56.4 \pm 8.9$ and $121.6 \pm$ $6.7, P=0.008$, respectively).

CONCLUSIONS: Our results suggest that hemodynamic factors may be associated with aneurysm recurrence among Pcom aneurysms. Velocity and flow rate in the Pcom may be a pretreatment prognostic factor for aneurysm recurrence after endovascular treatment.
\end{abstract}

Keywords: Computational fluid dynamics, intracranial aneurysm, recurrence; pressure, velocity, flow rate, endovascular treatment, coil

\section{Introduction}

The reported risk factors for recurrence of intracranial aneurysm following coil embolization include

\footnotetext{
${ }^{*}$ Corresponding author: Hiroyuki Takao, Division of Endovascular Neurosurgery, Department of Neurosurgery, The Jikei University School of Medicine, 3-25-8 Nishi-shinbashi, Minato-ku, Tokyo 105-8461, Japan. Tel.: +81 3 3433-3461; Fax: +81 33459 6412; E-mail: takao@jikei.ac.jp.
}

0928-7329/17/ $\$ 35.00$ (c) 2017 - IOS Press and the authors. All rights reserved

This article is published online with Open Access and distributed under the terms of the Creative Commons Attribution NonCommercial License (CC BY 4.0). 
Table 1

\begin{tabular}{lccc}
\multicolumn{4}{c}{ Morphological and treatment data for all included patients } \\
\hline Factors & Recurrence $(n=5)$ & Stable $(n=5)$ & $P$ value \\
\hline Dome size $(\mathrm{mm})$ & $6.63 \pm 1.38$ & $6.09 \pm 1.21$ & 0.548 \\
Neck size $(\mathrm{mm})$ & $5.18 \pm 1.84$ & $5.03 \pm 1.92$ & 1.000 \\
Pcom size $(\mathrm{mm})$ & $1.77 \pm 0.40$ & $2.06 \pm 0.21$ & 0.222 \\
Aneurysm volume $\left(\mathrm{mm}^{3}\right)$ & $97.4 \pm 46.8$ & $129.7 \pm 62.0$ & 0.841 \\
VER $(\%)$ & $26.2 \pm 6.60$ & $25.1 \pm 1.4$ & 0.841 \\
\hline
\end{tabular}

Pcom: posterior communicating artery; VER: volume embolization ratio.

large size, wide neck, and low coil packing ratio [1-6]. From comparisons of the aneurysm site, recurrences have been reported to be relatively common with posterior communicating artery (Pcom) aneurysms. The size of the Pcom has been investigated as a factor associated with recurrence but has not been found to be significant $[7,8]$.

Limits exist in the prediction of recurrence based on such anatomical factors, and hemodynamic factors therefore need to be investigated. Few such studies have been undertaken. Li et al. compared hemodynamics before and after treatment and reported that wall shear stress (WSS) of the neck was associated with recurrence following endovascular treatment, but pretreatment models revealed no significant factors [9]. Even though recurrence following treatment may be predictable, this does not enable precautionary measures to be taken; the ability to predict recurrence prior to treatment is thus crucial in clinical practice. We therefore used a pretreatment Pcom aneurysm model to investigate whether recurrence of Pcom aneurysm following coil embolization can be predicted from measurements of computational fluid dynamic (CFD) parameters.

\section{Materials and methods}

\subsection{Patient population}

This study was approved by the Jikei University School of Medicine Ethical Committee. We selected patients from among cases of coil embolization carried out at this facility from 2003 to 2011 in which angiographic examinations for recurrence 1 year after treatment showed an unruptured Pcom aneurysm. Cases with a maximum aneurysm diameter of 5-10 $\mathrm{mm}$ and volume embolization ratio (VER) $\geqslant 20 \%$ were selected. The local ethics committee approved the study, and informed consent was obtained from all participants prior to enrollment.

We defined aneurysm recurrence as the presence of opacification of the aneurysm sac on angiography $[7,10]$. Patients were divided according to this criterion into a recurrence group and a stable group $(n=5$, each) for analysis (Table 1). Patients with a Pcom $<1 \mathrm{~mm}$ in diameter or showing bifurcation at the aneurysm dome were excluded.

\section{2. $C F D$ model}

CFD modeling used images from pretreatment digital subtraction angiography that were converted into a triangulated surface using "Real Intage" (Cybernet Systems, Tokyo, Japan). An unstructured computational volumetric mesh was constructed from this triangulated surface. The mesh mainly comprised tetrahedrons with several layers of prism elements used near the wall surface to increase the analytic precision of the boundary layer. Pulsatile flow conditions were introduced as inflow conditions in the 


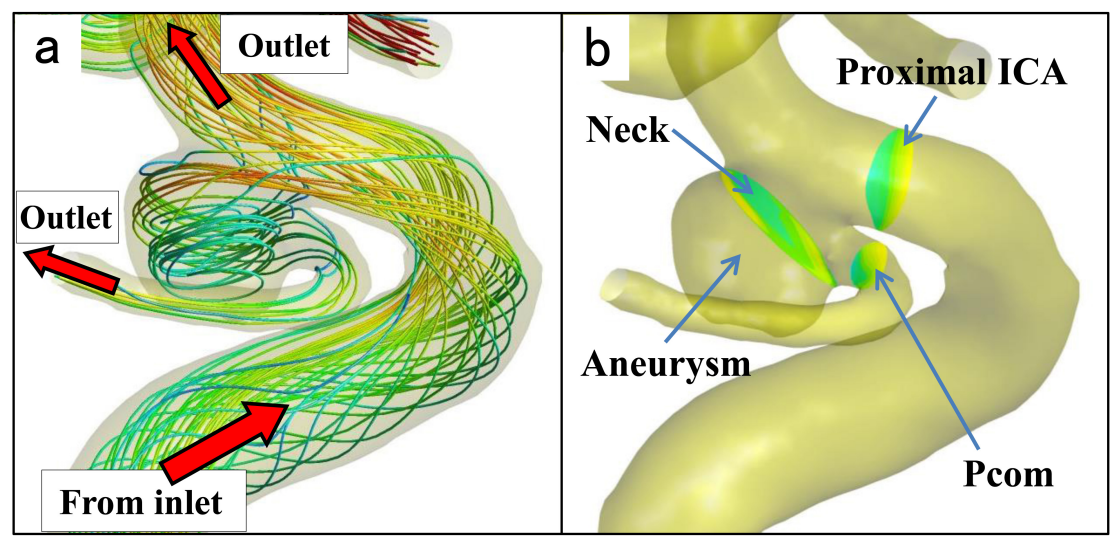

Fig. 1. Definition of vascular model. (a): The vascular and aneurysm model with streamlines showing a spatial relationship between inlet and outlets. The streamlines from the inlet, at the proximal site of the internal carotid artery (ICA), run to both the aneurysm and distal site of the ICA, which finally flow into outlet vessels, including the posterior communicating artery (Pcom). (b): The neck plane is defined as just distal to the Pcom bifurcation. The proximal ICA and Pcom planes are defined as $1 \mathrm{~mm}$ from the aneurysm.

internal carotid artery (ICA), with the average flow set at $0.254 \mathrm{~L} / \mathrm{min}$ and the pulse set at 70 beats per minute [11]. Under assumptions of pulsatile laminar flow, zero pressure at the blood vessel outlet, Newtonian fluid, and rigid blood vessel walls with nonslip conditions, Navier-Stokes equations were used to simulate blood flow on the computational mesh. Commercial software (ANSYS 12.1 ICEM CFD and CFX; ANSYS, Canonsburg, PA, USA) was used for both mesh generation and fluid simulation. Additional CFD modeling details were as described previously [11,12]. We collected information on the aneurysm characteristics from the vascular model, including dome size, neck size, and aneurysm volume, and adopted their maximum values. Coil volume was defined as $\pi(p / 2)^{2}$ multiplied by the coil length, where $p$ is the coil diameter. The VER was obtained by dividing the volume of all inserted coils by the aneurysm volume. We defined the aneurysm neck as the plane located just on the dome side of the Pcom origin because the surface of the coil mass was located near this plane. The proximal ICA and Pcom planes where static pressure and velocity were calculated were defined at $1 \mathrm{~mm}$ from the aneurysm (Fig. 1). Static pressure refers to the pressure exerted by blood, regardless of whether the fluid is in motion. Static pressure is frequently simply termed "pressure", and is distinguished from dynamic pressure, which represents fluid motion and is calculated from fluid density and velocity.

\subsection{Data analysis}

We calculated time-averaged static pressures and velocities for the proximal ICA, neck, and Pcom. Static pressures of the neck and Pcom were investigated as a pressure ratio:

$$
\text { Pressure ratio }=P_{\text {neck or } P \text { com }} / P_{\text {proximal ICA }}
$$

where $P$ (Pascal) is time-averaged static pressure. Pressure ratio is the time-averaged neck and Pcom pressure subtracted by the proximal ICA static pressure to give dimensionless values. Velocities of the neck and Pcom were investigated as dimensionless quantities by dividing by the proximal ICA velocity:

$$
\text { Velocity ratio }=V_{\text {neck or Pcom }} / V_{\text {proximal ICA }}
$$

where $V(\mathrm{~m} / \mathrm{s})$ is time-averaged velocity. Flow rate was calculated by multiplying the area of the neck and Pcom by the time-averaged velocity. 
Table 2

\begin{tabular}{lccc}
\multicolumn{4}{c}{ Hemodynamic factors related to aneurysm recurrence } \\
\hline & Recurrence $(n=5)$ & Stable $(n=5)$ & $P$ value \\
\hline Pressure ratio & & & \\
$\quad$ Neck & $-0.496 \pm 0.257$ & $-0.869 \pm 0.134$ & 0.151 \\
$\quad$ Pcom & $-1.891 \pm 0.637$ & $-3.112 \pm 0.302$ & 0.222 \\
Velocity ratio & & & \\
$\quad$ Neck & $0.591 \pm 0.017$ & $0.652 \pm 0.060$ & 0.421 \\
$\quad$ Pcom & $0.630 \pm 0.062$ & $0.926 \pm 0.051$ & 0.016 \\
Flow rate $\left(\mathrm{mL} \mathrm{min}{ }^{-1}\right)$ & & & \\
$\quad$ Neck & $492.4 \pm 144.7$ & $539.4 \pm 152.1$ & 0.841 \\
$\quad$ Pcom & $56.4 \pm 8.9$ & $121.6 \pm 6.7$ & 0.008 \\
Wall shear stress $(\mathrm{Pa})$ & $6.25 \pm 1.03$ & $8.39 \pm 0.64$ & 0.095 \\
\hline
\end{tabular}

Pcom: posterior communicating artery.

WSS varies in time (at different points in the heart cycle) and space (over the blood vessel wall). A spatial average of WSS was therefore computed over the aneurysmal wall in the measurement section followed by time averages along the simulation time course (two heart cycles). WSS is given by:

$$
\tau_{w}=\left.\mu \frac{\partial u}{\partial y}\right|_{y=0}
$$

where $y$ is the distance from the vessel surface, $u$ is the velocity of the fluid along the boundary, and $\mu$ is viscosity. Distance $y$ is constant, at $0.01 \mathrm{~mm}$ [13].

All statistical analyses were performed using IBM SPSS Statistics version 19.0 (SPSS, Chicago, IL). The nonparametric Mann-Whitney $U$ test was used to identify significant associations between hemodynamic parameters and recurrence. Statistical tests were conducted at the $\mathrm{P}<0.05$ significance level.

\section{Results}

No significant differences were found between the recurrence group and stable group in terms of dome size, neck size, Pcom size, aneurysm volume, or VER (Table 1). Pressure ratios at both the neck and Pcom tended to be slightly higher in the recurrence group than in the stable group, but these differences were not significant. Velocity ratios were significantly lower at the Pcom in the recurrence group $(0.630$ $\pm 0.062)$ than in the stable group $(0.926 \pm 0.051 ; P=0.016)$, but no significant difference was apparent at the neck. No significant difference in WSS was found between groups (Table 2).

Angiographic findings from representative cases are shown in Fig. 2, with the upper image showing a recurrent aneurysm and the lower showing a stable aneurysm. Depiction of the Pcom is slightly poor in the upper image, whereas the Pcom is depicted with abundant flow in the lower image. Although sufficient coil embolizations were performed with a VER $>20 \%$ in both cases, obvious recurrence due to coil compaction is only apparent in the upper image. Velocity ratio and flow rate of the Pcom were 0.423 and $28.2 \mathrm{~mL} \mathrm{~min}^{-1}$ in the upper case, and 0.793 and $111.8 \mathrm{~mL} \mathrm{~min}^{-1}$ in the lower case, respectively. The streamlines of both cases show that the Pcom velocity was lower in the recurrent case than in the stable case (Fig. 3).

\section{Discussion}

The streamline in Fig. 1a shows that the flow of the Pcom was derived from the aneurysm and that the flow around the aneurysm and Pcom is extremely complex and differs among individual patients. 

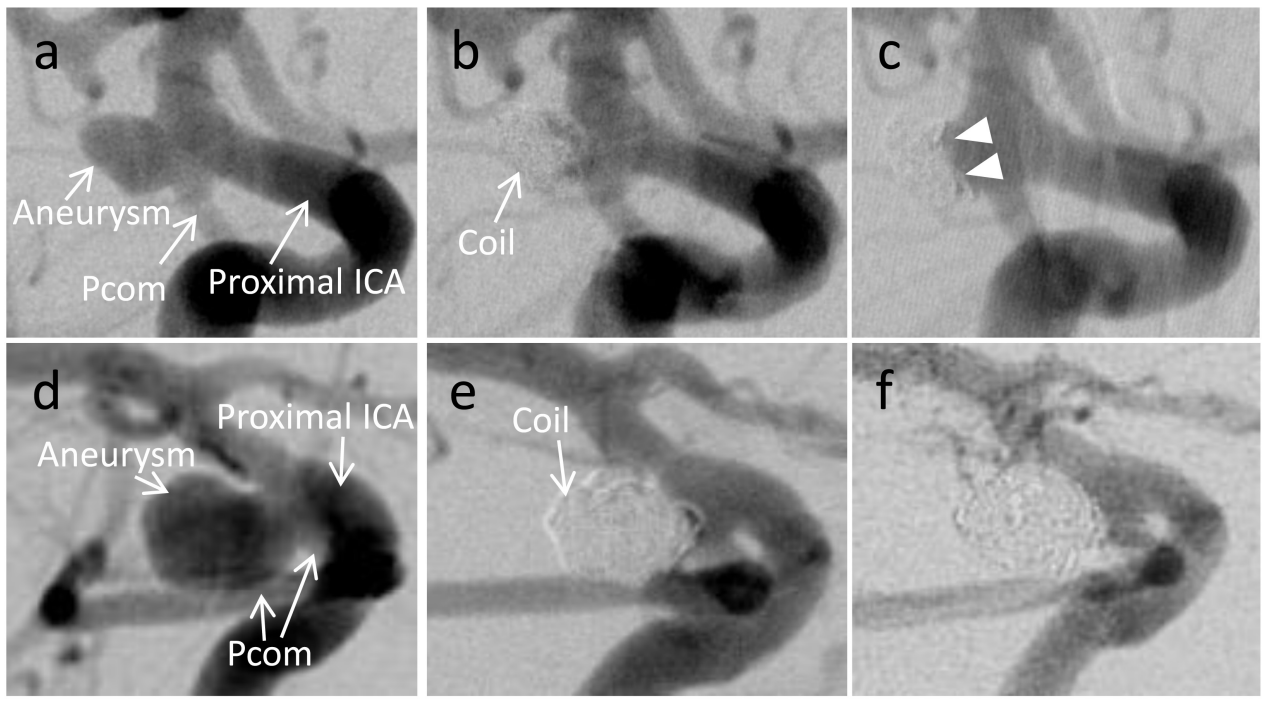

Fig. 2. Representative cases. (a): Pretreatment right internal carotid artery (ICA) angiography, lateral view, shows an unruptured posterior communicating artery (Pcom) aneurysm. Velocity ratio and flow rate of the Pcom are 0.397 and $47.9 \mathrm{~mL} \mathrm{~min}^{-1}$, respectively. (b): Right ICA angiography immediately after endovascular treatment shows sufficient occlusion with a small neck remnant, and the volume embolization ratio is $25.0 \%$. (c): Right ICA angiography 1 year after coil embolization reveals an obvious aneurysm recurrence due to coil compaction (arrowheads). (d): Right ICA angiography, right oblique view, before treatment demonstrates an unruptured Pcom aneurysm with abundant Pcom flow. Velocity ratio and flow rate of the Pcom are 0.793 and $111.8 \mathrm{~mL} \mathrm{~min}^{-1}$, respectively. (e): Post-treatment right ICA angiography shows the occluded aneurysm with a volume embolization ratio of $24.2 \%$. (f): ICA angiography 1 year after treatment reveals no aneurysm recurrence.

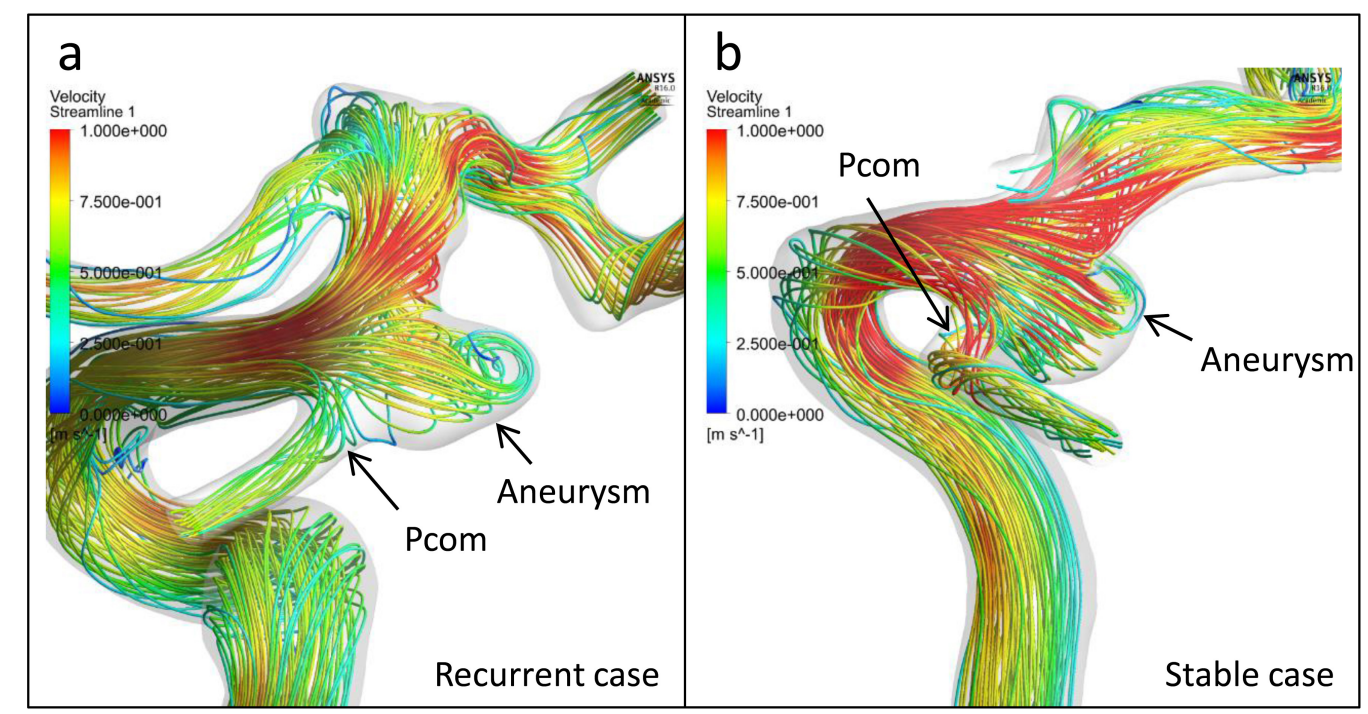

Fig. 3. Streamlines of the representative cases. (a): Streamlines of the pretreatment vascular model of the recurrent case in Fig. 2 show that the velocities of Pcom and ICA were almost 25 to $50 \mathrm{~cm} / \mathrm{s}$ and 75 to $100 \mathrm{~cm} / \mathrm{s}$, respectively. These results correspond to the velocity ratio of 0.397. (b): Streamlines of the representative stable case in Fig. 2 show that the velocities of Pcom and ICA were 75 to $100 \mathrm{~cm} / \mathrm{s}$ and almost $100 \mathrm{~cm} / \mathrm{s}$, respectively, which were consonant with the velocity ratio of 0.793 . 
We focused on the hemodynamics of the aneurysm and Pcom as predictive factors for aneurysmal recurrence after endovascular treatment. The fundamental hemodynamic factors are pressure (static pressure), velocity, and WSS. These factors were investigated as dimensionless quantities by dividing them by the values in the proximal ICA. For these reasons, we defined cut planes in the proximal ICA, neck, and Pcom.

The present study analyzed a group in which no significant differences were apparent between the recurrence group and stable group in aneurysm size, wide neck, or low coil packing ratio, all of which have previously been considered as factors potentially associated with recurrence after endovascular treatment [1-5,14-16]. As a VER $\leqslant 20 \%$ has been reported to be a significant factor for recurrence, the present study selected cases with VER $\geqslant 20 \%$ for analysis $[1,3,4,16]$. Technical difficulties in endovascular treatment, such as microcatheter instability and instability of the fit of the inserted coils, cause insufficient coil packing of aneurysms. We consider VER to be an index of technical difficulty and the occlusion state of aneurysms, and eliminated cases with a VER $<20 \%$. In addition, aneurysms of 5$10 \mathrm{~mm}$ in diameter were selected to eliminate the aneurysm size factor. The effects of recurrence factors other than hemodynamic factors were thus extremely small.

In the present study, relative blood flow velocity and flow rate in the Pcom to inflow vessels (proximal ICA) were significantly lower in the recurrence group than in the stable group. Clinically, some cases showed rapid Pcom washout during angiography and others showed slow washout. The velocity decreases when blood flow at high velocity collides with a vascular wall, and the energy is converted into static pressure. Velocity and static pressure are thus believed to have complementary actions in fluids [17]. A tendency toward high static pressure is believed to arise in areas where the velocity is low, and in the present study, the Pcom velocity was significantly lower in the recurrence group than in the stable group. Similarly, although the difference was not significant, pressure tended to be slightly higher in the recurrence group. Whether this slightly higher pressure increases after endovascular treatment and influences recurrence remains unclear. The significantly low velocities and flow rates in the Pcom in the recurrence group mean that the washout of the Pcom is slow. The detailed mechanisms of aneurysm recurrence in terms of the effects of Pcom stagnation remain unclear. A rheological investigation of this issue using a post-coil placement model is needed.

With regard to the relationship between recurrence after coil embolization and WSS, Li et al. found no significant difference in WSS between the recurrence group and stable group in the pretreatment model [9]. The present study identified no significant difference in WSS on the aneurysmal wall between the recurrence group and the stable group, consistent with the findings of $\mathrm{Li}$ et al.

This investigation performed analyses based on a pre-treatment model, and is therefore useful in screening for aneurysms which have the risk of recurrence. The ability to predict a high risk of recurrence prior to treatment would make it possible to consider combining coil embolization with stents, which could be expected to bring about hemodynamic alterations [18], or changing the therapeutic strategy to clipping surgery. Rheological examination before treatment is therefore useful.

Limitations of the present study include the retrospective design and the small number of patients, which limit the statistical power, and these preliminary findings need further validations with large samples. Additionally, this report did not evaluate hemodynamics using a post-treatment model. There are a few recent reports that analyzed hemodynamics with a post-treatment aneurysm model [19]. Cases in which the Pcom was not depicted on angiography and cases in which the aneurysm was $\leqslant 1 \mathrm{~mm}$ in diameter were not examined. In fluid dynamics analysis, we made many assumptions regarding pulsatile laminar flow, zero pressure at the blood vessel outlet, and Newtonian fluid behaviors in the NavierStokes equation. Boundary conditions were uniform across all cases, and vessel walls were assumed to 
be rigid, whereas in reality, deformation may occur during the cardiac cycle. The boundary pressure of the Pcom was uniformly set to zero, irrespective of whether the Pcom actually received some flow from the proximal posterior cerebral artery.

\section{Conclusion}

Our results suggest that pretreatment hemodynamic factors are associated with the recurrence of Pcom aneurysms. Among these factors, the velocity and flow rate of the Pcom may allow prediction of recanalization following treatment.

\section{Conflict of interest}

Y.M and T.I. have received honoraria from Stryker Japan. Y.M. has received honoraria from ASAHI INTECC Co., Ltd. Y.M. and H.T. were partially supported by SIEMENS K.K. Japan with a grant provided to our academic institution (Grant No. 35993-00211563). H.T. was supported by NTT DOCOMO, Inc., with a donation provided to our academic institution outside the submitted work.

\section{References}

[1] Kawanabe Y, Sadato A, Taki W, Hashimoto N. Endovascular occlusion of intracranial aneurysms with Guglielmi detachable coils: correlation between coil packing density and coil compaction. Acta Neurochirurgica. 2001; 143: 451-5.

[2] Sluzewski M, van Rooij WJ, Slob MJ, Bescos JO, Slump CH, Wijnalda D. Relation between aneurysm volume, packing, and compaction in 145 cerebral aneurysms treated with coils. Radiology. 2004; 231: 653-8.

[3] Tamatani S, Ito Y, Abe H, Koike T, Takeuchi S, Tanaka R. Evaluation of the stability of aneurysms after embolization using detachable coils: correlation between stability of aneurysms and embolized volume of aneurysms. AJNR American Journal of Neuroradiology. 2002; 23: 762-7.

[4] Uchiyama N, Kida S, Nomura M, Hasegawa M, Yamashima T, Yamashita J, et al. Significance of volume embolization ratio as a predictor of recanalization on endovascular treatment of cerebral aneurysms with guglielmi detachable coils. Interventional Neuroradiology: Journal of Peritherapeutic Neuroradiology, Surgical Procedures and Related Neurosciences. 2006; Suppl 159-63.

[5] Yagi K, Satoh K, Satomi J, Matsubara S, Nagahiro S. Evaluation of aneurysm stability after endovascular embolization with Guglielmi detachable coils: correlation between long-term stability and volume embolization ratio. Neurologia Medico-Chirurgica. 2005; 45: 561-5; discussion 5-6.

[6] Takao H, Ishibashi T, Saguchi T, Arakawa H, Ebara M, Irie K, et al. Validation and initial application of a semiautomatic aneurysm measurement software: a tool for assessing volumetric packing attenuation. AJNR American Journal of Neuroradiology. 2014; 35: 721-6.

[7] Raymond J, Guilbert F, Weill A, Georganos SA, Juravsky L, Lambert A, et al. Long-term angiographic recurrences after selective endovascular treatment of aneurysms with detachable coils. Stroke; A Journal of Cerebral Circulation. 2003; 34: 1398-403.

[8] Songsaeng D, Geibprasert S, Willinsky R, Tymianski M, TerBrugge KG, Krings T. Impact of anatomical variations of the circle of Willis on the incidence of aneurysms and their recurrence rate following endovascular treatment. Clinical Radiology. 2010; 65: 895-901.

[9] Li C, Wang S, Chen J, Yu H, Zhang Y, Jiang F, et al. Influence of hemodynamics on recanalization of totally occluded intracranial aneurysms: a patient-specific computational fluid dynamic simulation study. Journal of Neurosurgery. 2012; 117: 276-83.

[10] Roy D, Milot G, Raymond J. Endovascular treatment of unruptured aneurysms. Stroke; a Journal of Cerebral Circulation. 2001; 32: 1998-2004.

[11] Takao H, Murayama Y, Otsuka S, Qian Y, Mohamed A, Masuda S, et al. Hemodynamic differences between unruptured and ruptured intracranial aneurysms during observation. Stroke; A Journal of Cerebral Circulation. 2012; 43: 1436-9.

[12] Ford MD, Alperin N, Lee SH, Holdsworth DW, Steinman DA. Characterization of volumetric flow rate waveforms in the normal internal carotid and vertebral arteries. Physiological Measurement. 2005; 26: 477-88. 
[13] Qian Y, Takao H, Umezu M, Murayama Y. Risk analysis of unruptured aneurysms using computational fluid dynamics technology: preliminary results. AJNR American Journal of Neuroradiology. 2011; 32: 1948-55.

[14] Ries T, Siemonsen S, Thomalla G, Grzyska U, Zeumer H, Fiehler J. Long-term follow-up of cerebral aneurysms after endovascular therapy prediction and outcome of retreatment. AJNR American Journal of Neuroradiology. 2007; 28: 1755-61.

[15] Mortimer AM, Marsh H, Klimczak K, Joshi D, Barton H, Nelson RJ, et al. Is long-term follow-up of adequately coiloccluded ruptured cerebral aneurysms always necessary? A single-center study of recurrences after endovascular treatment. Journal of Neurointerventional Surgery. 2015; 7: 373-9.

[16] Leng B, Zheng Y, Ren J, Xu Q, Tian Y, Xu F. Endovascular treatment of intracranial aneurysms with detachable coils: correlation between aneurysm volume, packing, and angiographic recurrence. Journal of Neurointerventional Surgery. 2014; 6: 595-9.

[17] Shojima M, Oshima M, Takagi K, Torii R, Nagata K, Shirouzu I, et al. Role of the bloodstream impacting force and the local pressure elevation in the rupture of cerebral aneurysms. Stroke; A Journal of Cerebral Circulation. 2005; 36: 1933-8.

[18] Jeong W, Han MH, Rhee K. The hemodynamic alterations induced by the vascular angular deformation in stent-assisted coiling of bifurcation aneurysms. Computers in Biology and Medicine. 2014; 53: 1-8.

[19] Nair P, Chong BW, Indahlastari A, Ryan J, Workman C, Haithem Babiker M, et al. Hemodynamic characterization of geometric cerebral aneurysm templates treated with embolic coils. Journal of Biomechanical Engineering. 2016; 138: 021011. 